\title{
Proteomic identification of differentially expressed proteins in the anoxic rice coleoptile
}

\author{
Irfan Sadiq ${ }^{a}$, Francesca Fanucchi $^{\mathrm{b}}$, Eleonora Paparelli ${ }^{\mathrm{a}}$, Emanuele Alpi $^{\mathrm{c}}$, Angela Bachi $^{\mathrm{c}}$, Amedeo Alpi $^{\mathrm{b}}$, \\ Pierdomenico Perata ${ }^{a, *}$ \\ a Plant Lab, Institute of Life Sciences, Scuola Superiore Sant'Anna, Pisa, Italy \\ ${ }^{\mathrm{b}}$ Department of Crop Plant Biology, University of Pisa, Pisa, Italy \\ ${ }^{\mathrm{c}}$ Biomolecular Mass Spectrometry Unit, San Raffaele Scientific Institute, Milan, Italy
}

\section{A R T I C L E I N F O}

\section{Article history:}

Received 13 June 2011

Received in revised form 21 July 2011

Accepted 21 July 2011

\section{Keywords:}

Anoxia

Coleoptile

Proteomics

Rice

\begin{abstract}
A B S T R A C T
Rice is the staple food for more than fifty percent of the world's population, and is therefore an important crop. However, its production is hindered by several biotic and abiotic stresses. Although rice is the only crop that can germinate even in the complete absence of oxygen (i.e. anoxia), flooding (low oxygen) is one of the major causes of reduced rice production. Rice germination under anoxia is characterized by the elongation of the coleoptile, but leaf growth is hampered. In this work, a comparative proteomic approach was used to detect and identify differentially expressed proteins in the anoxic rice coleoptile compared to the aerobic coleoptile. Thirty-one spots were successfully identified by MALDI-TOF MS analysis. The majority of the identified proteins were related to stress responses and redox metabolism. The expression levels of twenty-three proteins and their respective mRNAs were analyzed in a time course experiment.

(C) 2011 Elsevier GmbH. All rights reserved.
\end{abstract}

\section{Introduction}

With the growth in world population, there has been an increasing demand for food. However, due to the environmental stresses that negatively affect plant growth and productivity, crop production is not increasing at the same rate. Environmental stress means economic hardship for farmers and may be life-threatening for regions that depend on subsistence farming. In order to increase crop yields and to expand cultivated areas, increased plant tolerance to environmental stress is therefore essential. Plants are sessile organisms that must develop different defense strategies against unfavorable conditions. These strategies are often mediated by changes in gene expression leading to the production of proteins that are thought to be possibly involved in tolerance

Abbreviations: 2-DE, two-dimensional gel electrophoresis; ADF4, actin depolymerising factor 4; ADH, alcohol dehydrogenase; ALDH, aldehyde dehydrogenase; ANPs, anaerobic proteins; APX, ascorbate peroxidise; $C B B$, Comassie brilliant blue; CBL, Calcineurin B-like protein; CIPKs, CBL-interacting protein kinases; GSTU, glutathione S-transferase; IEF, isoelectric focusing; IPG, immobilized pH gradient; MS, mass spectrometry; PDC, pyruvate decarboxylase; ROS, reactive oxygen species; sHSPs, small heat shock proteins; SOD, superoxide dismutase; TUBA1, tubulin $\alpha-1$ chain; Usp, universal stress protein.

* Corresponding author at: Plant Lab, Institute of Life Sciences, Scuola Superiore Sant'Anna, Piazza Martiri della Libertá 33, 56127 Pisa, Italy. Tel.: +39 0502211585 ; fax: +390502211581 .

E-mail address: p.perata@sssup.it (P. Perata).
(Timperio et al., 2008). One of the most important stresses in crops is flooding, which decreases oxygen and light supply to the submerged parts of plants, negatively affecting crop productivity (Bailey-Serres and Voesenek, 2010). Because gas diffusion in water is very slow, the submerged plant experiences drastic changes in oxygen, $\mathrm{CO}_{2}$ availability as well as ethylene entrapment (BaileySerres and Voesenek, 2010). Oxygen diffuses ten thousand times slower in water compared to air (Armstrong, 1980). In submerged plants, leaves, as a result of photosynthesis, may have relatively high oxygen content, while roots are often very hypoxic (BaileySerres and Voesenek, 2010).

Rice (Oryza sativa) is the staple food of more than $50 \%$ of the world's population. The ability of rice seeds to germinate under anoxic conditions allows the direct sowing of rice, which is more economical that transplanting (Perata and Alpi, 1993; Magneschi and Perata, 2009). Indeed, in the tropics, rice sowing is usually performed by distributing seeds in paddy fields that are submerged by water (Yamauchi et al., 2000).

During rice germination, the coleoptile grows much faster when submerged compared to the aerobic coleoptile, enabling the seedling to reach the water surface and thus escape from the unfavorable low oxygen environment (Magneschi and Perata, 2009). This makes study of the rice coleoptile of interest for both plant biology and agronomy. No root and primary leaf growth is observed in rice seedlings germinated under anoxia, a trait that helps to save energy before the coleoptile tip reaches the water surface. Under low oxygen stress, a metabolic adjustment takes place to 
cope with this unfavorable condition. One of the major changes is in the carbohydrate metabolism (Geigenberger, 2003; Licausi and Perata, 2009). Rice has the ability to degrade starch even under anoxia, due to the presence of amylolytic enzyme activities, which are either absent or inactive in other cereals such as wheat and barley (anoxic intolerant cereals) (Perata et al., 1998). These results in the use of the starchy reserves, with concomitant ATP production through the fermentative pathway, thus enabling rice grains to germinate even in the complete absence of oxygen (Guglielminetti et al., 1995).

A microarray analysis of gene expression in anoxic rice coleoptiles revealed a wide range of differentially expressed genes (Lasanthi-Kudahettige et al., 2007), indicating that anoxia could involve the cross-talk of pathways involved in different stresses from anoxia, such as heat. Indeed, heat acclimation can increase the anoxia tolerance of Arabidopsis seedlings (Banti et al., 2010). These studies shed light on some of the processes activated during low oxygen stress, but do not answer all of the pertinent questions.

Proteomic studies on plants exposed to low oxygen stress have shown that, in addition to the expression of classical anaerobic proteins (ANPs), there is also expression of proteins related to other mechanisms, such as reactive oxygen species (ROS) scavengers and protein synthesis in wheat (Kong et al., 2010), heat stress response in soybean (Hashiguchi et al., 2009), intracellular trafficking in maize (Chang et al., 2000), gibberellin biosynthesis, heme biosynthesis, and cell wall degradation in tomatoes (Ahsan et al., 2007).

Despite the extensive knowledge available on the effects of anoxia at a transcriptomic level, no adequate work has yet been done at a proteomic level in rice, with the exception of three reports by Mujer et al. (1993), Millar et al. (2004), and Huang et al. (2005). In the study by Mujer et al. (1993), rice seedlings were grown under anoxia immediately after seed imbibition; however, their identification was based on the immunoblotting of a very limited number of known ANPs. Millar et al. (2004) restricted their study to mitochondrial proteins extracted from rice seedlings grown for six days under anoxia and then transferred to air for one day, while Huang et al. (2005) grew seedlings in air and then exposed them to anoxia.

In this work, rice seeds were germinated in anoxia and the anoxic coleoptile proteome was compared to the aerobic coleoptile. We used anoxia because, under these experimentally controlled conditions, it is possible to identify changes that are due to the lack of oxygen only, uncoupling the metabolic and molecular adjustment in the coleoptile in response to anoxia from other consequences of submergence, such as ethylene entrapment by water (Bailey-Serres and Voesenek, 2010). Our aim was to identify differentially expressed proteins under anoxia and to follow their expression levels at different stages of coleoptile elongation.

\section{Materials and methods}

\section{Seeds sterilization and growth}

Rice (Oryza Sativa, cv. Arborio) seeds were surface sterilized using a diluted sodium hypochlorite solution $(1.7 \%, v / v)$ and washed with distilled sterile water several times. Seeds were germinated at $28^{\circ} \mathrm{C}$ in the dark in Petri dishes containing five sterile filter paper disks, soaked with $8 \mathrm{~mL}$ of distilled sterile water. For the anoxic treatments, seeds were germinated inside an anoxic chamber (Anaerobic System model 1025; Forma Scientific) after seed imbibition as described by Lasanthi-Kudahettige et al. (2007). The primary leaf and root did not elongate under anoxia. We selected four-day-old seedlings to perform the initial protein profiling, since at this growth stage both the aerobic and anoxic coleoptiles were large enough to enable them to be rapidly dissected from the seedlings. In the time course experiment, coleoptiles were collected from 3-, 4-, 5- and 6-day-old grown rice seedlings. Coleoptiles were dissected and immediately frozen in liquid nitrogen, and stored at $-80^{\circ} \mathrm{C}$ for protein and total RNA extraction.

\section{Protein extraction and quantification}

Soluble proteins were extracted from rice coleoptiles according to Yang et al. (2007), with some modifications. Coleoptiles were ground in liquid nitrogen and homogenized with $1 \mathrm{~mL}$ of extraction buffer ( $5 \mathrm{M}$ urea, $2 \mathrm{M}$ thiourea, $40 \mathrm{mM}$ Tris- $\mathrm{HCl}, 2 \%$, CHAPS, $50 \mathrm{mM}$ DTT). The homogenates were centrifuged for $15 \mathrm{~min}$ at $15,000 \times \mathrm{g}$. Supernatants were precipitated using TCA $(15 \%, v / v)$ containing $0.007 \% \beta$-mercaptoethanol in acetone at $-20^{\circ} \mathrm{C}$ for $2 \mathrm{~h}$ and then at $4{ }^{\circ} \mathrm{C}$ for a minimum of $2 \mathrm{~h}$. Samples were centrifuged at $4{ }^{\circ} \mathrm{C}$ for $15 \mathrm{~min}$ at $14,000 \times \mathrm{g}$, supernatants were discarded and pellets were washed twice with ice cold acetone containing $0.007 \% \beta$ mercaptoethanol. Pellets were dissolved in a rehydration buffer (5 M urea, $2 \mathrm{M}$ thiourea, 4\%, CHAPS, $40 \mathrm{mM}$ DTT). Protein quantification was performed using a Bradford-based assay kit assay (Bio Rad Hercules, CA), using bovine serum albumin as a standard.

\section{$2 D$ electrophoresis}

Isoelectric focusing (IEF) of total proteins was performed using $18 \mathrm{~cm}$-long immobilized $\mathrm{pH}$ gradient (IPG) strips, $\mathrm{pH}$ 3-10 non linear and $\mathrm{pH} 4-7$. The protein sample was mixed with a rehydration buffer, $0.5 \%$ IPG buffer ( $\mathrm{v} / \mathrm{v}$ ) of respective $\mathrm{pH}$ range and $0.002 \%$ bromophenol blue to a final volume of $340 \mu \mathrm{L}$ and loaded onto the IEF strips. Samples were loaded onto IPG strips by passive rehydration. For analytical gels, performed to obtain the silver-stained protein maps, $100 \mu \mathrm{g}$ of the protein sample was loaded. Instead a $500 \mu \mathrm{g}$ sample was loaded for the preparative gels stained with mass spectrometry (MS) compatible silver nitrate and $1 \mathrm{mg}$ of the sample was loaded for Coomassie brilliant blue (CBB) staining. IEF was carried out at $200 \mathrm{~V}$ for $3 \mathrm{~h}, 1000 \mathrm{~V}$ for $1 \mathrm{~h}, 2000 \mathrm{~V}$ for $1 \mathrm{~h}, 3500 \mathrm{~V}$ for $1 \mathrm{~h}$ and $35 \mathrm{kVh}$ using the Multiphore II system (Amersham Pharmacia Biotech). Before running the second dimension SDS-PAGE, IPG strips were equilibrated twice in an equilibration buffer ( $6 \mathrm{M}$ urea, $30 \%$ glycerol (v/v), $50 \mathrm{mM}$ Tris- $\mathrm{HCl}, 2 \%)$ SDS for $15 \mathrm{~min}$. The first equilibration was done using $1.2 \%$ DTT $(\mathrm{w} / \mathrm{v})$ in an equilibration buffer, while in the second equilibration, DTT was replaced by $1.5 \%$ iodoacetamide $(\mathrm{w} / \mathrm{v})$. SDS-PAGE was performed using $12.5 \%$ polyacrylamide gels at $15^{\circ} \mathrm{C}$ using a BioRad Protean II XI $(20 \mathrm{~cm} \times 20 \mathrm{~cm})$ vertical gel electrophoresis chamber. After completion of the electrophoresis, gels were fixed and stained.

The analytical gels were stained for image analysis with silver nitrate as described by Oakley et al. (1980), while for the MS analysis, the preparative gels were stained with CBB according to the manufacturer's instructions, and MS compatible silver nitrate staining was performed as described by Mortz et al. (2001). Three independent biological replicates, each with four technical replicates were run for the analytical gels. In the time course experiment, three independent biological replicates were run, each with two technical replicates.

\section{Gel image and statistical analysis}

The silver stained analytical gels were scanned at 300 dpi resolution and saved as TIF images for image analysis. The two best gels were selected out of each biological replicate set of four. Spot detection and quantification were performed using Nonlinear Progenesis Same Spots software (Nonlinear Dynamics, Newcastle upon Tyne, UK, version 4.0). Images were subjected to manual as well as automatic alignment. Both aerobic and anoxic pairwise comparisons were performed and fold values as well as p-values for all spots 

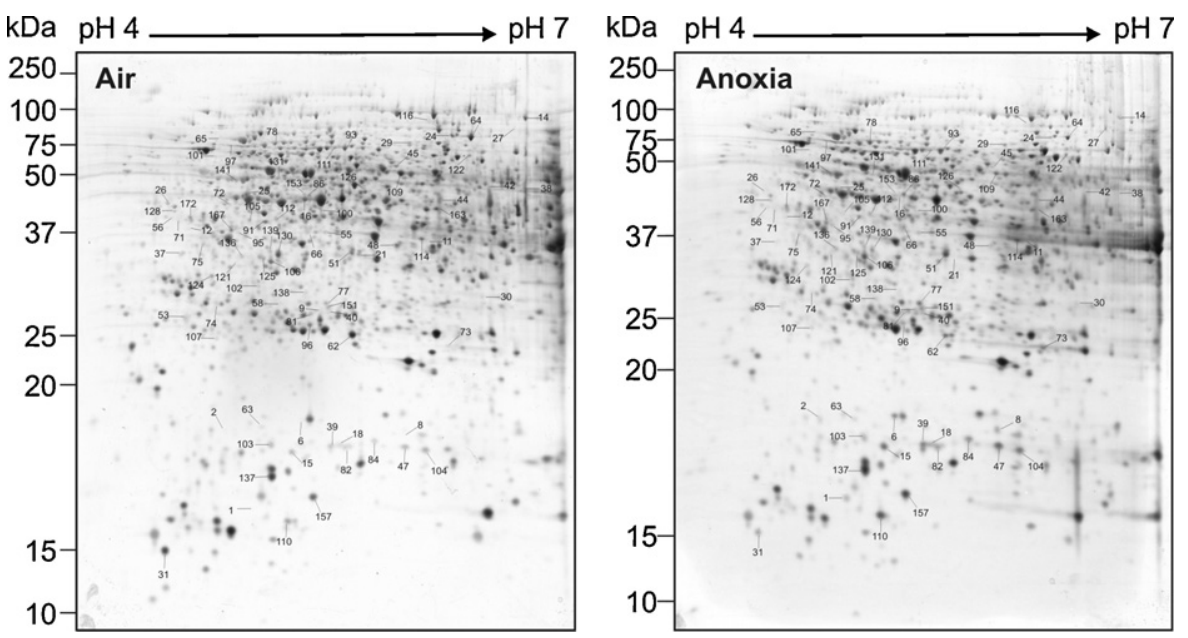

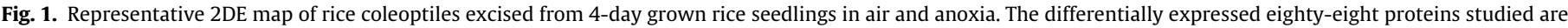
numbered on the gels. Gels were stained with silver nitrate and image analysis was performed using Nonlinear Progenesis Same Spot software.

were calculated using the software mentioned above. All spots were pre-filtered and manually checked before applying the statistical analysis of variance criteria $(p<0.05$ and fold $>1.5)$. Statistical analysis of continuous data (spot normalized volume) was evaluated using the non-parametric Kruskal-Wallis test (Glover and Mitchell, 2002). The spots that showed significant and reproducible changes were selected for MS analysis.

In the time course experiment, silver stained analytical gels were scanned and analyzed using the software described to observe the protein expression behavior of selective protein spots at different time points. Three independent biological replicates were analyzed, each with two technical replicates.

\section{Trypsin digestion and protein identification by MALDI-TOF MS}

Protein spots of interest were excised from gels, reduced, alkylated, and in-gel digested overnight with bovine trypsin (Roche Diagnostics Corp.) as previously described (Shevchenko et al., 1996). Aliquots of digested samples $(1 \mu \mathrm{L})$ were used for MALDI analysis by the dried-droplet technique, using $\alpha$-cyano-4hydroxycinnamic acid as a matrix. The tryptic digests were desalted and concentrated when needed, before sample loading on the target. Mass spectra were obtained using a MALDI-TOF Voyager DE-STR from Applied Biosystems/MDS Sciex. Ions were generated by irradiation with a pulsed nitrogen laser $(337 \mathrm{~nm}$ UV, pulse duration $3 \mathrm{~ns}$, pulse rate $3 \mathrm{~Hz}$ ) and positive ions were accelerated and detected in the reflector mode. Instrument settings were as follows: accelerating $20,000 \mathrm{~V}$, grid $64 \%$, guide wire $0 \%$, delay time $200 \mathrm{~ns}$, shots/spectrum 100, mass range 750-4000 Da and low mass gate $700 \mathrm{Da}$. Spectra were internally calibrated using trypsin autolysis products and acquired via Voyager Control Panel 5.10 (Applied Biosystems). Once acquired, spectra were processed with Data Explorer 4.0 (Applied Biosystems).

MALDI-TOF data led to extracted and manually curated peptide monoisotopic peak lists (deprived from trypsin and the most common keratin signals) which were searched for in the target database as detailed below. Peak lists were also searched for (via Mascot Daemon 2.2.2), first in a custom (trypsin and common keratins) contaminant database derived from the publicly available CRAP repository. Unmatched signals were then searched for in the most recent UniProtKB database available at the time of the analysis, either with $O$. sativa as a taxonomy filter or without (version 2010_07, 11656695 sequences, 144347 O. sativa sequences), with an in-house Mascot Server 2.2.07 (Matrix Science, London, UK) (Perkins et al., 1999). Mass tolerance for monoisotopic data on peptides was set to $50 \mathrm{ppm}$. Searches were performed with trypsin specificity, alkylation of cysteine by carbamidomethylation, and oxidation of methionine as fixed and variable modifications, respectively; two missed cleavages were allowed for trypsin specificity; a significance threshold of $p<0.05$ was set for the probability based Mascot Mowse Score.

\section{Total RNA extraction and real time PCR}

Total RNA was extracted from coleoptiles of 3-, 4-, 5- and 6-day-old grown rice seedlings in air and anoxia as previously described (Perata et al., 1997) with a minor modification (omission of aurintricarboxylic acid) to make the protocol compatible with the subsequent PCR procedures. Electrophoresis using a $1 \%$ agarose gel was performed for all RNA samples to check for RNA integrity, followed by spectrophotometric quantification. Contaminating DNA was removed using a TURBO DNA-free kit (Ambion, http://www.ambion.com/). RNA was then reverse-transcribed using an iScript ${ }^{\mathrm{TM}}$ cDNA Synthesis kit (BioRad Laboratories, http://www.bio-rad.com). Genes corresponding to our proteins of interest were identified by searching for the relative Accession Number (Table 1) in the UniProtKB and Gramene databases (http://www.uniprot.org; http://www.gramene.org), and confirmed by BLAST. Expression analysis of these genes was performed by real-time PCR using an ABI Prism 7300 sequence detection system (Applied Biosystems). Quantitative PCR was performed using $20 \mathrm{ng} \mathrm{cDNA}^{\mathrm{B}}$ and $\mathrm{iQ}^{\mathrm{TM}}$ SYBR $^{\circledR}$ Green Supermix (BioRad Laboratories), according to the manufacturer's instructions. Expressions of Actin1 were used as endogenous controls. Relative expression levels were calculated using Genorm (http://medgen.ugent.be/ jvdesomp/genorm). For a list of the primers used see Table S2.

\section{Results and discussion}

\section{The anoxic proteome of rice coleoptiles}

A comparison of the coleoptile proteome using a $\mathrm{pH}$ range 3-10 revealed the presence of the majority of spots in a $\mathrm{pH}$ range from 4 to 7 (Fig. S1). This pH range (4-7) was used for both the image analysis and protein identification and enables 697 protein spots to be detected (Fig. 1). Of these, several showed varied protein amounts under anoxia (Fig. 2A).

Eighty-eight protein spots (see Table 1) were classified as differentially expressed by computer-assisted two-dimensional gel 
Table 1

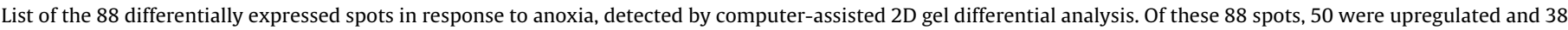
were downregulated compared to the aerobic control. Data are means of three independent biological replicates, each with two technical replicates $( \pm \mathrm{SD}$ ).

\begin{tabular}{|c|c|c|c|c|c|}
\hline \multirow[t]{2}{*}{ Spot No. } & \multicolumn{2}{|c|}{ Average normalized volumes } & \multirow[t]{2}{*}{ Up/down regulated under anoxia } & \multirow[t]{2}{*}{ Fold change } & \multirow[t]{2}{*}{ Anova $(P)$} \\
\hline & Air & Anoxia & & & \\
\hline 1 & $809,887.074$ & $13,387,237.86$ & Upregulated & 16.5 & $3.71 \mathrm{E}-05$ \\
\hline 2 & $697,491.048$ & $6,454,747.607$ & Upregulated & 9.3 & $2.23 \mathrm{E}-05$ \\
\hline 6 & $2,093,713.578$ & $10,282,758.14$ & Upregulated & 4.9 & $1.51 \mathrm{E}-04$ \\
\hline 8 & $1,425,023.523$ & $5,865,497.814$ & Upregulated & 4.1 & 0.002 \\
\hline 9 & $2,062,329.974$ & $8,179,294.715$ & Upregulated & 4 & $8.96 \mathrm{E}-05$ \\
\hline 11 & $3,497,341.193$ & $12,811,668.07$ & Upregulated & 3.7 & $2.90 \mathrm{E}-04$ \\
\hline 12 & $1,243,173.646$ & $4,440,932.227$ & Upregulated & 3.6 & 0.009 \\
\hline 14 & $11,134,687.21$ & $3,206,564.674$ & Downregulated & -3.5 & $1.06 \mathrm{E}-04$ \\
\hline 15 & $7,678,815.653$ & $25,923,112.07$ & Upregulated & 3.4 & $2.93 \mathrm{E}-04$ \\
\hline 16 & $7,224,776.399$ & $2,149,196.316$ & Downregulated & -3.4 & $8.38 \mathrm{E}-04$ \\
\hline 18 & $2,425,251.507$ & $7,756,404.819$ & Upregulated & 3.2 & $7.34 \mathrm{E}-04$ \\
\hline 21 & $5,756,297.889$ & $1,828,252.981$ & Downregulated & -3.1 & 0.001 \\
\hline 24 & $6,159,896.075$ & $2,058,463.669$ & Downregulated & -3 & 0.003 \\
\hline 25 & $6,176,000.983$ & $2,106,993.013$ & Downregulated & -2.9 & $1.08 \mathrm{E}-04$ \\
\hline 26 & $2,385,483.215$ & $819,545.408$ & Downregulated & -2.9 & 0.002 \\
\hline 27 & $1,803,315.284$ & $624,398.938$ & Downregulated & -2.9 & 0.013 \\
\hline 29 & $5,514,657.578$ & $2,025,267.11$ & Downregulated & -2.7 & 0.001 \\
\hline 30 & $1,823,852.826$ & $670,619.955$ & Downregulated & -2.7 & 0.001 \\
\hline 31 & $30,418,561.08$ & $11,259,279.05$ & Downregulated & -2.7 & 0.002 \\
\hline 37 & $3,611,450.684$ & $1,394,578.579$ & Downregulated & -2.6 & 0.01 \\
\hline 38 & $15,245,727.45$ & $5,890,943.773$ & Downregulated & -2.6 & 0.003 \\
\hline 39 & $10,417,752.33$ & $26,906,752.81$ & Upregulated & 2.6 & 0.005 \\
\hline 40 & $1,619,991.445$ & $4,179,302.284$ & Upregulated & 2.6 & $1.98 \mathrm{E}-04$ \\
\hline 42 & $2,000,220.737$ & $785,990.497$ & Downregulated & -2.5 & 0.008 \\
\hline 44 & $9,112,906.543$ & $3,610,193.317$ & Downregulated & -2.5 & $5.53 \mathrm{E}-04$ \\
\hline 45 & $3,219,968.976$ & $1,276,754.329$ & Downregulated & -2.5 & 0.006 \\
\hline 47 & $9,750,986.149$ & $24,419,397.79$ & Upregulated & 2.5 & 0.002 \\
\hline 48 & $10,756,341.83$ & $4,296,755.745$ & Downregulated & -2.5 & 0.004 \\
\hline 50 & $22,923,033.32$ & $9,298,967.994$ & Downregulated & -2.5 & 0.016 \\
\hline 51 & $14,389,763.77$ & $35,074,269.43$ & Upregulated & 2.4 & 0.005 \\
\hline 53 & $6,901,579.1$ & $2,910,492.28$ & Downregulated & -2.4 & $8.38 \mathrm{E}-04$ \\
\hline 55 & $2,416,064.321$ & $5,725,941.264$ & Upregulated & 2.4 & 0.014 \\
\hline 56 & $731,881.273$ & $1,717,026.509$ & Upregulated & 2.3 & 0.008 \\
\hline 58 & $1,417,327.656$ & $3,304,112.279$ & Upregulated & 2.3 & $4.62 \mathrm{E}-04$ \\
\hline 62 & $35,178,293.6$ & $15,481,911.33$ & Downregulated & -2.3 & $4.92 \mathrm{E}-04$ \\
\hline 63 & $1,442,453.961$ & $3,255,153.968$ & Upregulated & 2.3 & $1.28 \mathrm{E}-04$ \\
\hline 64 & $21,573,952.39$ & $9,574,372.451$ & Downregulated & -2.3 & 0.013 \\
\hline 65 & $1,179,093.11$ & $2,633,717.341$ & Upregulated & 2.2 & 0.004 \\
\hline 66 & $4,309,783.451$ & $9,543,263.253$ & Upregulated & 2.2 & 0.003 \\
\hline 71 & $476,876.711$ & $1,021,940.022$ & Upregulated & 2.1 & 0.007 \\
\hline 72 & $1,281,453.287$ & $2,741,179.283$ & Upregulated & 2.1 & 0.012 \\
\hline 73 & $10,300,920.06$ & $22,009,666.74$ & Upregulated & 2.1 & 0.009 \\
\hline 74 & $2,377,634.493$ & $1,118,357.942$ & Downregulated & -2.1 & 0.007 \\
\hline 75 & $2,859,272.234$ & $1,348,655.065$ & Downregulated & -2.1 & 0.005 \\
\hline 77 & $4,018,152.481$ & $8,449,523.963$ & Upregulated & 2.1 & 0.008 \\
\hline 78 & $6,720,247.643$ & $14,121,288.68$ & Upregulated & 2.1 & 0.009 \\
\hline 81 & $5,231,732.159$ & $10,955,185.05$ & Upregulated & 2.1 & 0.007 \\
\hline 82 & $7,073,896.846$ & $14,742,957.3$ & Upregulated & 2.1 & 0.002 \\
\hline 84 & $5,928,866.678$ & $12,303,676.01$ & Upregulated & 2.1 & 0.003 \\
\hline 86 & $3,438,677.888$ & 7,090,899.107 & Upregulated & 2.1 & 0.009 \\
\hline 91 & $6,755,760.913$ & $3,330,838.259$ & Downregulated & -2 & 0.001 \\
\hline 93 & $13,395,835.78$ & $26,860,516.35$ & Upregulated & 2 & 0.008 \\
\hline 96 & $6,881,488.375$ & $3,533,731.683$ & Downregulated & -1.9 & 0.008 \\
\hline 97 & $2,673,583.442$ & $5,163,594.869$ & Upregulated & 1.9 & 0.015 \\
\hline 100 & $1,658,378.314$ & $874,472.317$ & Downregulated & -1.9 & 0.01 \\
\hline 101 & $1,592,540.892$ & $848,938.939$ & Downregulated & -1.9 & 0.011 \\
\hline 102 & $4,682,937.711$ & $8,736,977.292$ & Upregulated & 1.9 & 0.006 \\
\hline 103 & $8,079,279.437$ & $15,000,904.34$ & Upregulated & 1.9 & 0.01 \\
\hline 104 & $10,895,828.18$ & $20,185,483.54$ & Upregulated & 1.9 & 0.008 \\
\hline 105 & $3,033,016.28$ & $1,649,344.549$ & Downregulated & -1.8 & 0.009 \\
\hline 106 & $13,887,516.82$ & $25,262,406.19$ & Upregulated & 1.8 & 0.012 \\
\hline 107 & $1,688,294.244$ & $3,059,186.138$ & Upregulated & 1.8 & 0.008 \\
\hline 109 & $30,429,653.81$ & $16,818,484.77$ & Downregulated & -1.8 & 0.007 \\
\hline 110 & $24,057,811.47$ & $43,303,170.06$ & Upregulated & 1.8 & 0.008 \\
\hline 111 & $2,744,010.038$ & $4,923,743.641$ & Upregulated & 1.8 & 0.008 \\
\hline 112 & $3,796,960.538$ & $6,762,507.21$ & Upregulated & 1.8 & 0.008 \\
\hline 114 & $8,996,813.426$ & $15,810,775.53$ & Upregulated & 1.8 & 0.006 \\
\hline 116 & $1,422,843.201$ & $811,651.811$ & Downregulated & -1.8 & 0.004 \\
\hline 121 & $763,768.962$ & $1,317,673.049$ & Upregulated & 1.7 & 0.011 \\
\hline 122 & $3,289,314.111$ & $1,921,674.619$ & Downregulated & -1.7 & 0.012 \\
\hline 124 & $5,837,238.587$ & $3,420,239.099$ & Downregulated & -1.7 & 0.008 \\
\hline 125 & $2,715,452.031$ & $4,620,751.879$ & Upregulated & 1.7 & 0.004 \\
\hline 126 & $1,676,296.796$ & $991,997.911$ & Downregulated & -1.7 & 0.014 \\
\hline
\end{tabular}


Table 1 (Continued)

\begin{tabular}{|c|c|c|c|c|c|}
\hline \multirow[t]{2}{*}{ Spot No. } & \multicolumn{2}{|c|}{ Average normalized volumes } & \multirow[t]{2}{*}{ Up/down regulated under anoxia } & \multirow[t]{2}{*}{ Fold change } & \multirow[t]{2}{*}{ Anova $(P)$} \\
\hline & Air & Anoxia & & & \\
\hline 128 & $1,148,833.916$ & $1,926,313.481$ & Upregulated & 1.7 & 0.01 \\
\hline 130 & $21,608,938.63$ & $13,015,000.59$ & Downregulated & -1.7 & 0.008 \\
\hline 131 & $3,198,037.965$ & $1,927,492.039$ & Downregulated & -1.7 & 0.016 \\
\hline 136 & $4,078,336.13$ & $6,683,013.981$ & Upregulated & 1.6 & $1.15 \mathrm{E}-05$ \\
\hline 137 & $40,546,040.62$ & $66,171,905.49$ & Upregulated & 1.6 & 0.015 \\
\hline 138 & $1,337,063.635$ & $821,379.51$ & Downregulated & -1.6 & 0.015 \\
\hline 139 & $2,648,071.763$ & $4,298,426.466$ & Upregulated & 1.6 & 0.01 \\
\hline 141 & $4,749,689.549$ & $7,648,222.499$ & Upregulated & 1.6 & 0.009 \\
\hline 151 & $5,060,813.35$ & $7,911,604.135$ & Upregulated & 1.6 & 0.014 \\
\hline 153 & $2,568,287.808$ & $1,651,791.083$ & Downregulated & -1.6 & 0.016 \\
\hline 157 & $23,908,830.13$ & $36,192,291.71$ & Upregulated & 1.5 & 0.001 \\
\hline 163 & $12,014,503.63$ & $8,014,267.204$ & Downregulated & -1.5 & $3.25 \mathrm{E}-05$ \\
\hline 167 & $8,342,022.254$ & $5,587,792.056$ & Downregulated & -1.5 & 0.012 \\
\hline 172 & $9,574,491.721$ & $13,938,700.71$ & Upregulated & 1.5 & 0.005 \\
\hline
\end{tabular}

electrophoresis (2-DE) gel differential analysis (Fig. 1). Of these, 50 spots were upregulated and 38 were downregulated compared to the aerobic control (Table 1; Fig. 2B). Forty-nine spots were excised from preparative gels for mass spectrometry (MS) analysis. Thirtyone spots were identified successfully using MALDI-TOF MS and twenty-four univocally corresponded to a single protein (Table 2; Fig. 2B), whereas the remaining seven corresponded to more than one (Table S1).

The identified proteins were classified into eleven different functional categories, based on the UniProtKB database classification: redox homeostasis (17\%), carbohydrate metabolism (20\%), Small heat shock protein (sHSP) and chaperon (10\%), signal transduction (3\%), protein synthesis (5\%), protein degradation $(2 \%)$, amino acid synthesis (2\%), cell structure related (5\%), hormones (10\%), secondary metabolism (2\%), and others (24\%) (Fig. S2).

Having identified the spots listed in Table 2, we performed a time course experiment aimed at analyzing both the protein and the
mRNA profiles of 23 differentially regulated proteins. The protein expression patterns and their respective transcripts are shown in Fig. S3.

\section{Proteins involved in the stress response}

Under anoxia, plants switch from aerobic to anaerobic respiration (Licausi and Perata, 2009). Several genes encoding for carbohydrate metabolism and fermentative enzymes are induced in the anoxic rice coleoptile (Umeda and Uchimiya, 1994; LasanthiKudahettige et al., 2007). Ethanolic fermentation is an important pathway when a lack of oxygen hampers mitochondrial ATP production and it is often related to the survival of plants under low oxygen stress. Ethanolic fermentation requires two enzymes, namely alcohol dehydrogenase (ADH) and pyruvate decarboxylase (PDC) (Bailey-Serres and Voesenek, 2010).

Table 2

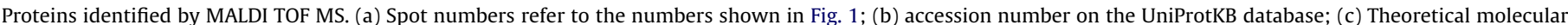

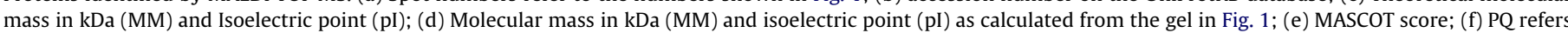

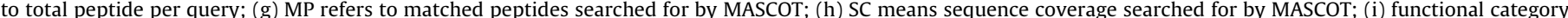

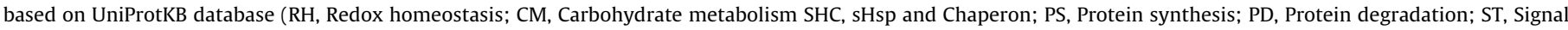
transduction; AS, Amino acid synthesis; CS, Cell structure; HR, Hormones; SM, Secondary metabolism; OT, others.

\begin{tabular}{|c|c|c|c|c|c|c|c|c|c|c|}
\hline Spot No. (a) & $\begin{array}{l}\text { Protein } \\
\text { name }\end{array}$ & $\begin{array}{l}\text { UniProtKB } \\
\text { Acc. No. (b) }\end{array}$ & $\begin{array}{l}\text { Theo.MM } \\
\mathrm{kDa} / \mathrm{pI}(\mathrm{c})\end{array}$ & $\begin{array}{l}\text { Exp.MM } \\
\mathrm{kDa} / \mathrm{pI}(\mathrm{d})\end{array}$ & $\begin{array}{l}\text { Mascot } \\
\text { score (e) }\end{array}$ & $P Q(f)$ & $\mathrm{MP}(\mathrm{g})$ & SC \% (h) & $\begin{array}{l}\text { Funct. } \\
\text { Cat. (i) }\end{array}$ & $E$ value \\
\hline 2 & Universal stress protein & Q10MK7 & $19.2 / 5.01$ & $19.0 / 4.93$ & 72 & 15 & 5 & 31.70 & OT & $8.70 \mathrm{E}-03$ \\
\hline 6 & $24.1 \mathrm{kDa}$ heat shock protein, mitochondrial & Q6Z7V2 & $24.08 / 6.88$ & $20.0 / 5.48$ & 196 & 29 & 16 & 56.80 & $\mathrm{SHC}$ & $3.60 \mathrm{E}-15$ \\
\hline 14 & Putative CCT chaperonin gamma subunit & Q5Z6U5 & $61.31 / 6.23$ & $90.0 / 6.87$ & 157 & 31 & 17 & 34.20 & SHC & $2.90 \mathrm{E}-11$ \\
\hline 15 & $18.0 \mathrm{kDa}$ class II heat shock protein & Q5VRY1 & $18.13 / 5.61$ & $18.0 / 5.39$ & 108 & 13 & 8 & 39.2 & SHC & $2.30 \mathrm{E}-06$ \\
\hline 16 & IAA-amino acid hydrolase ILR1-like 3 & Q851L5 & $44.07 / 5.44$ & $50.0 / 5.48$ & 172 & 36 & 16 & 46.8 & HR & $9.10 \mathrm{E}-13$ \\
\hline 21 & Putative isoflavone reductase & Q9FTN5 & $33.48 / 5.69$ & $35.0 / 5.8$ & 131 & 30 & 12 & 48.4 & SM & $1.10 \mathrm{E}-08$ \\
\hline 42 & Putative uncharacterized protein & Q9AX68 & $40.11 / 7.64$ & $53.0 / 6.61$ & 104 & 27 & 10 & 30 & OT & $5.70 \mathrm{E}-06$ \\
\hline 45 & Mitochondrial aldehyde dehydrogenase ALDH2a & Q9LRI6 & $59.15 / 6.24$ & $60.0 / 6.03$ & 98 & 58 & 16 & 33.1 & $\mathrm{CM}$ & $2.60 \mathrm{E}-05$ \\
\hline 47 & Calcineurin B-like protein 5 (CBL5) & Q3HRP2 & $25.13 / 4.79$ & $18.0 / 6.10$ & 71 & 12 & 5 & 20.2 & ST & $1.30 \mathrm{E}-02$ \\
\hline 48 & $\begin{array}{l}\text { OSIGBa0152K17.4 protein (NADH-dependent, } \\
\text { oxidoreductase, aldo/keto reductase family } \\
\text { protein) }\end{array}$ & Q01J83 & $36.25 / 5.81$ & $37 / 6.03$ & 171 & 23 & 13 & 38.1 & $\mathrm{RH}$ & $1.10 \mathrm{E}-12$ \\
\hline 51 & Glucose and ribitol dehydrogenase homolog & Q75KH3 & $32.47 / 5.76$ & $36 / 5.75$ & 71 & 21 & 7 & 26 & $\mathrm{CM}$ & $2.80 \mathrm{E}-04$ \\
\hline 62 & Probable glutathione S-transferase GSTU6 & Q06398 & $25.69 / 5.82$ & $25 / 5.76$ & 98 & 33 & 9 & 42.8 & $\mathrm{RH}$ & $2.30 \mathrm{E}-05$ \\
\hline 73 & Proteasome subunit beta type-1 (PBF1) & 064464 & $24.60 / 6.43$ & $24 / 6.36$ & 105 & 11 & 7 & 38.5 & PD & $4.60 \mathrm{E}-06$ \\
\hline 74 & L-Ascorbate peroxidase 2, cytosolic (APX2) & Q9FE01 & $27.21 / 5.21$ & $29 / 4.92$ & 57 & 15 & 5 & 29.5 & RH & $5.90 \mathrm{E}-03$ \\
\hline 86 & IAA-amino acid hydrolase ILR1-like 3 & Q851L5 & $44.07 / 5.44$ & $51 / 5.50$ & 96 & 30 & 10 & 26.9 & $\mathrm{HR}$ & $3.50 \mathrm{E}-05$ \\
\hline 93 & Pyruvate decarboxylase isozyme 2 (PDC2) & Q10MW3 & $65.76 / 5.53$ & $70 / 5.71$ & 132 & 43 & 16 & 29.1 & $\mathrm{CM}$ & $9.10 \mathrm{E}-09$ \\
\hline 96 & Putative glutathione S-transferase GST27 & Q69LE6 & $25.12 / 5.53$ & $28 / 5.50$ & 81 & 25 & 7 & 34.5 & $\mathrm{RH}$ & $1.10 \mathrm{E}-03$ \\
\hline 109 & Aldehyde dehydrogenase ALDH2b & Q9FRX7 & $56.61 / 6.33$ & $59 / 6.08$ & 223 & 45 & 23 & 35.5 & $\mathrm{CM}$ & $7.20 \mathrm{E}-18$ \\
\hline 110 & Glyoxalase family protein, putative & Q0DT04 & $15.04 / 5.54$ & $14 / 5.38$ & 104 & 17 & 7 & 60.4 & OT & $5.70 \mathrm{E}-06$ \\
\hline 111 & $\begin{array}{l}\text { 2,3-Bisphosphoglycerate-independent } \\
\text { phosphoglycerate mutase, putative }\end{array}$ & Q5QMK7 & $60.98 / 5.42$ & $70 / 5.66$ & 134 & 25 & 14 & 33.1 & $\mathrm{CM}$ & $5.70 \mathrm{E}-09$ \\
\hline 131 & Enolase & B9G3A0 & $49.19 / 5.97$ & $59 / 5.29$ & 163 & 23 & 14 & 38.5 & $\mathrm{CM}$ & $7.20 \mathrm{E}-12$ \\
\hline 137 & 40 S ribosomal protein $\mathrm{S} 12$ & Q8H2J8 & $15.11 / 5.33$ & $16 / 5.27$ & 87 & 24 & 7 & 57.2 & PS & $3.20 \mathrm{E}-04$ \\
\hline 141 & Tubulin alpha- 1 chain, putative, expressed & Q10DN0 & $46.53 / 4.89$ & $56 / 5.01$ & 170 & 28 & 15 & 48.1 & CS & $1.40 \mathrm{E}-12$ \\
\hline 157 & Actin-depolymerizing factor 4 (ADF4) & Q84TB3 & $16.05 / 5.72$ & $15 / 5.56$ & 114 & 16 & 9 & 70.5 & CS & $5.70 \mathrm{E}-07$ \\
\hline
\end{tabular}




\section{A}

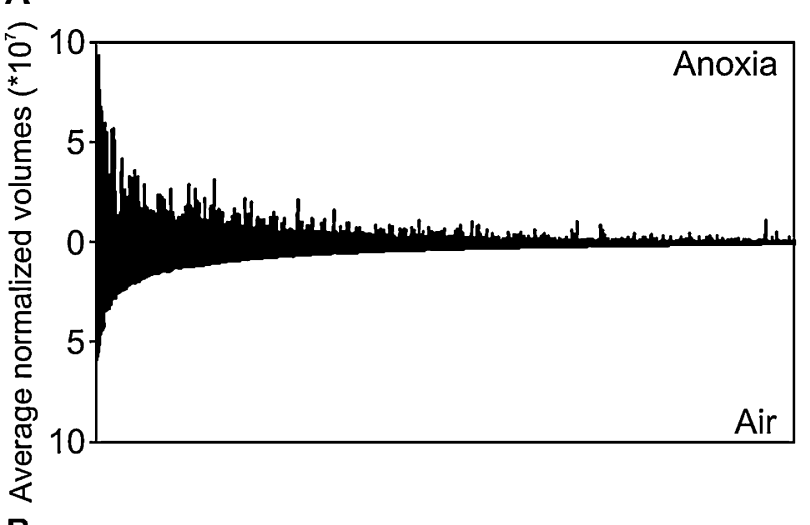

B

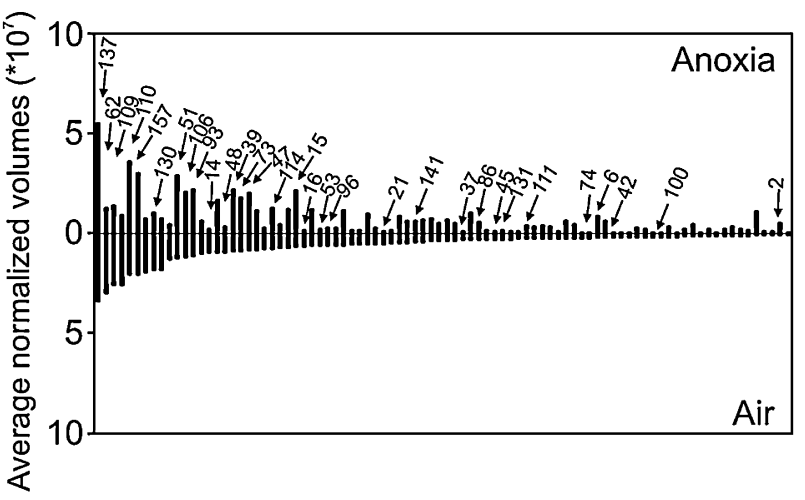

Fig. 2. Effect of anoxic treatment on the pattern of protein synthesis in 4-day-grown rice coleoptiles. (A) Average normalized volumes of 697 protein spots detected on 2DE gels (air and anoxia). Spots are ordered from the highest to the lowest normalized volumes in air. (B) Average normalized volumes of the 88 protein spots showing differential expressions in response to anoxia. The arrows indicate the 31 spots which were identified by MALDI-TOF MS analysis and correspond to those listed in Table 1. Data are the means of three independent biological replicates.

Spot 93 was identified as pyruvate decarboxylase (PDC2), which converts pyruvate into acetaldehyde and $\mathrm{CO}_{2}$. Anoxia induced PDC2 both at the protein and transcript levels (Fig. 3), which is likely to divert the fermentative pathway towards the production of acetaldehyde and ethanol rather than towards the lactic acid or alanine fermentative pathways (Tadege et al., 1998).

Aldehyde dehydrogenase (ALDH) converts the toxic acetaldehyde into acetate (Perata and Alpi, 1991). Two mitochondrial aldehyde genes are represented in the rice genome, $A L D H 2 a$ and $A L D H 2 b$. We identified aldehyde dehydrogenase2a (ALDH2a, spot 45 ) and aldehyde dehydrogenase2b (ALDH2b, spot 109). The expression of ALDH2a showed no significant changes under anoxia, except at day 4, when it was downregulated. Instead, the ALDH2a transcript was strongly upregulated under anoxia (Fig. 3). ALDH2b protein and mRNA levels were both low under anoxia (Fig. 3). In fact, $A L D H 2 b$ is known to be downregulated by anoxia (Tsuji et al., 2003), while ALDH2a mRNA has been shown to strongly accumulate under oxygen deprivation (Nakazono et al., 2000). Tsuji et al. (2003) detected increased ALDH2a mRNA levels under submergence, but no corresponding change in protein expression levels, which is in agreement with our results (spot 45, Fig. 3). As postulated by Nakazono et al. (2000), the conversion of acetaldehyde to acetate by ALDH consumes NAD, which could potentially block glycolysis. This may explain why the ALDH2a protein did not accumulate despite a marked increase in its mRNA level (Fig. 3). On the other hand, ALDH2a is localized in the mitochondria, which should reduce the risk of competition for NAD between ALDH activity and glycolysis (Nakazono et al., 2000). The low level of ALDH2a protein, together with the high induction of its mRNA under anoxia, suggests increased ALDH2a levels during post-anoxia in order to metabolize acetaldehyde. This hypothesis deserves further investigation.

Spot number 47 was identified as Calcineurin B-like protein 5 (CBL5), upregulated under anoxic stress both at the protein and mRNA levels (Fig. 3). CBLs are proteins that contain the EF motif, a common calcium binding domain. In plants, calcium $\left(\mathrm{Ca}^{2+}\right)$ levels increase in response to various stresses, including anoxia (Subbaiah et al., 1994). This change in cellular $\mathrm{Ca}^{2+}$ levels is recognized by calcium sensor proteins with $\mathrm{Ca}^{2+}$ binding motifs. CBL proteins bind $\mathrm{Ca}^{2+}$, undergo conformational changes, and interact with CBL-interacting protein kinases (CIPKs) to activate downstream signaling processes. The rice genome contains 10 CBLs and 30 CIPKs, which interact with each other to form specific pairs (Kolukisaoglu et al., 2004). A recent study by Lee et al. (2009) showed that CIPK15 plays an important role in the survival of rice plants under low oxygen stress. The identity of CBL protein(s) that interact with CIPK15 is unknown, and CBL5, which we demonstrated to be upregulated under anoxia, represents an excellent candidate. Experiments are underway to verify this hypothesis.

Spot 2 was identified as a universal stress protein (Usp). The transcript for this protein, encoded by Os03g0305400 was highly induced by anoxia, with an increase also at the protein level (Fig. 3). The expression of another universal stress protein (OsUsp1; Os07g0673400) was observed transiently during submergence treatment and may play a role in ethylene-mediated stress adaptation in rice (Sauter et al., 2002). The function of Usps in plants, however, is largely unknown.

sHSPs are not only expressed under heat stress, but also in response to other stresses in different plant species, such as in response to anoxia and osmotic stresses in Arabidopsis (Banti et al., 2010; Sun et al., 2001), salinity in maize (Hamilton and Heckathorn, 2001), and cold and oxidative stress in rice (Sarkar et al., 2009; Lee et al., 2000). sHSPs function as molecular chaperons (Sun et al., 2002) and can prevent the stress-dependent aggregation of proteins under stress conditions to maintain their functionality (Lee et al., 1997).

Spots 6 and 15 were identified as HSPs, namely $24.1 \mathrm{kDa}$ mitochondrial heat shock protein and $18.0 \mathrm{kDa}$ class II heat shock protein, respectively. Both proteins were upregulated under anoxia. The mRNA encoding the $24.1 \mathrm{kDa}$ mitochondrial heat shock protein was high under anoxia, in line with the high level of its corresponding protein. However, the transcript corresponding to the $18.0 \mathrm{kDa}$ class II heat shock protein (spot 15) was downregulated at days 4 and 5 , while at days 3 and 4 it showed no change in response to anoxia (Fig. 3). However, since the protein level of this SHSP was already high at day 3 , it is tempting to speculate that the protein accumulation observed resulted in the expression of the gene at an earlier stage of the coleoptile development.

Arabidopsis seedlings subjected to a mild heat pretreatment can better tolerate anoxia (Loreti et al., 2005; Banti et al., 2008) showing that the heat and anoxic stress response pathways partly overlap. Remarkably, in Arabidopsis HSP, protein accumulation is not observed under anoxia, where only the HSP mRNAs accumulate (Banti et al., 2010). Instead, the rice coleoptile displays sHSPs protein accumulation under anoxia (Fig. 3), which suggests that HSPs play an active role in conferring tolerance to anoxia.

\section{Redox homeostasis}

ROS are often produced in plants in response to biotic and abiotic stresses. Although ROS may act as important signaling molecules involved in acclimation to stress, these compounds are also potentially toxic to the plant. To mitigate the negative effects of ROS, enzymes acting as ROS scavengers are produced, such as ascorbate 

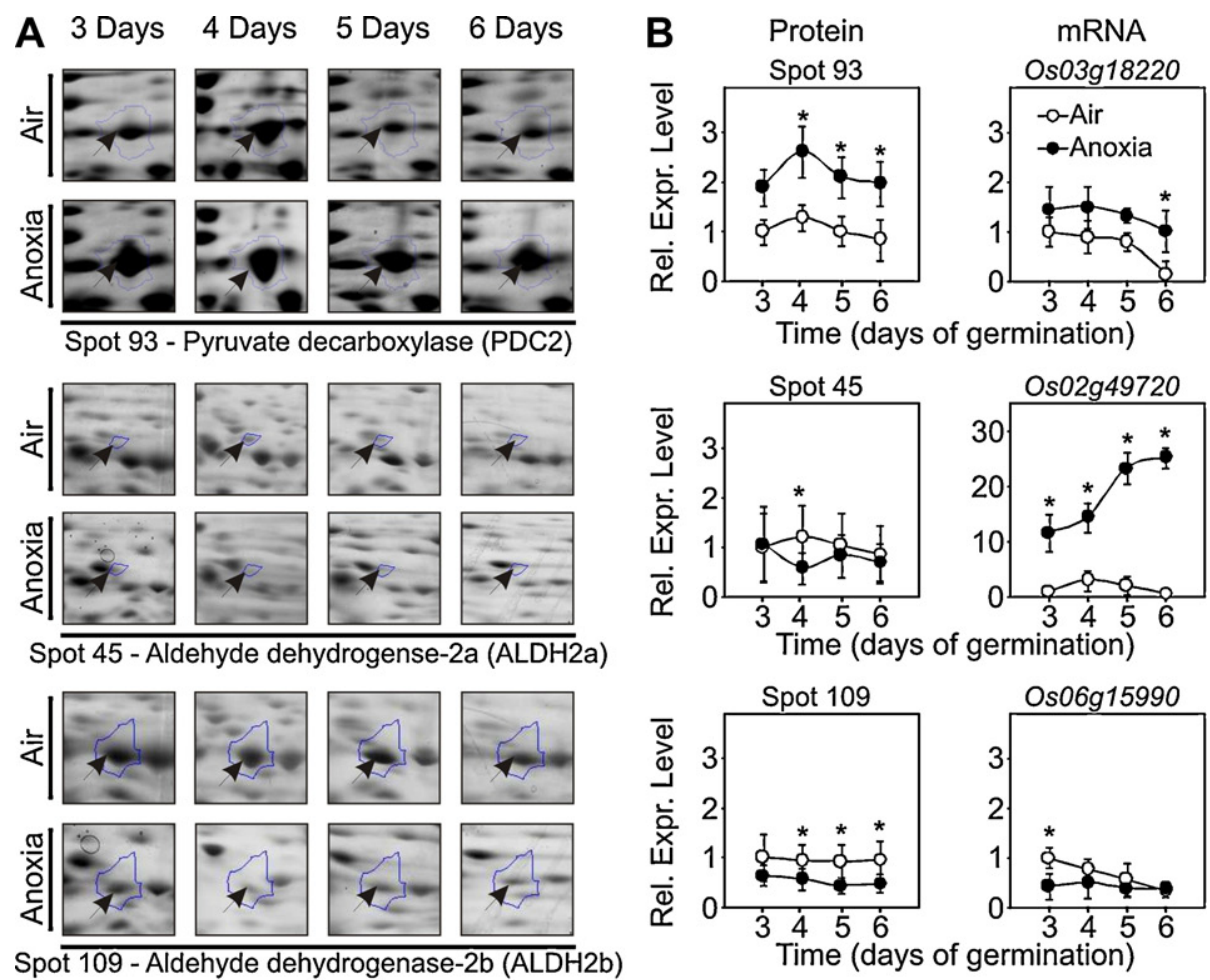

Spot 109 - Aldehyde dehydrogenase-2b (ALDH2b)

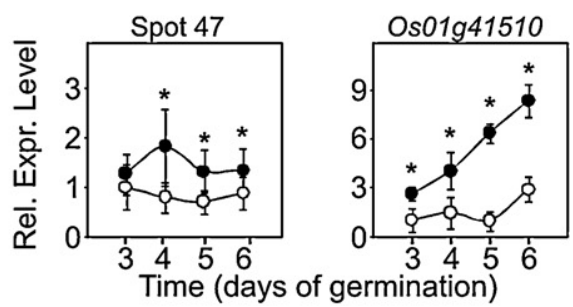

Spot 47 - Calcineurin B-like protein 5 (CBL5)
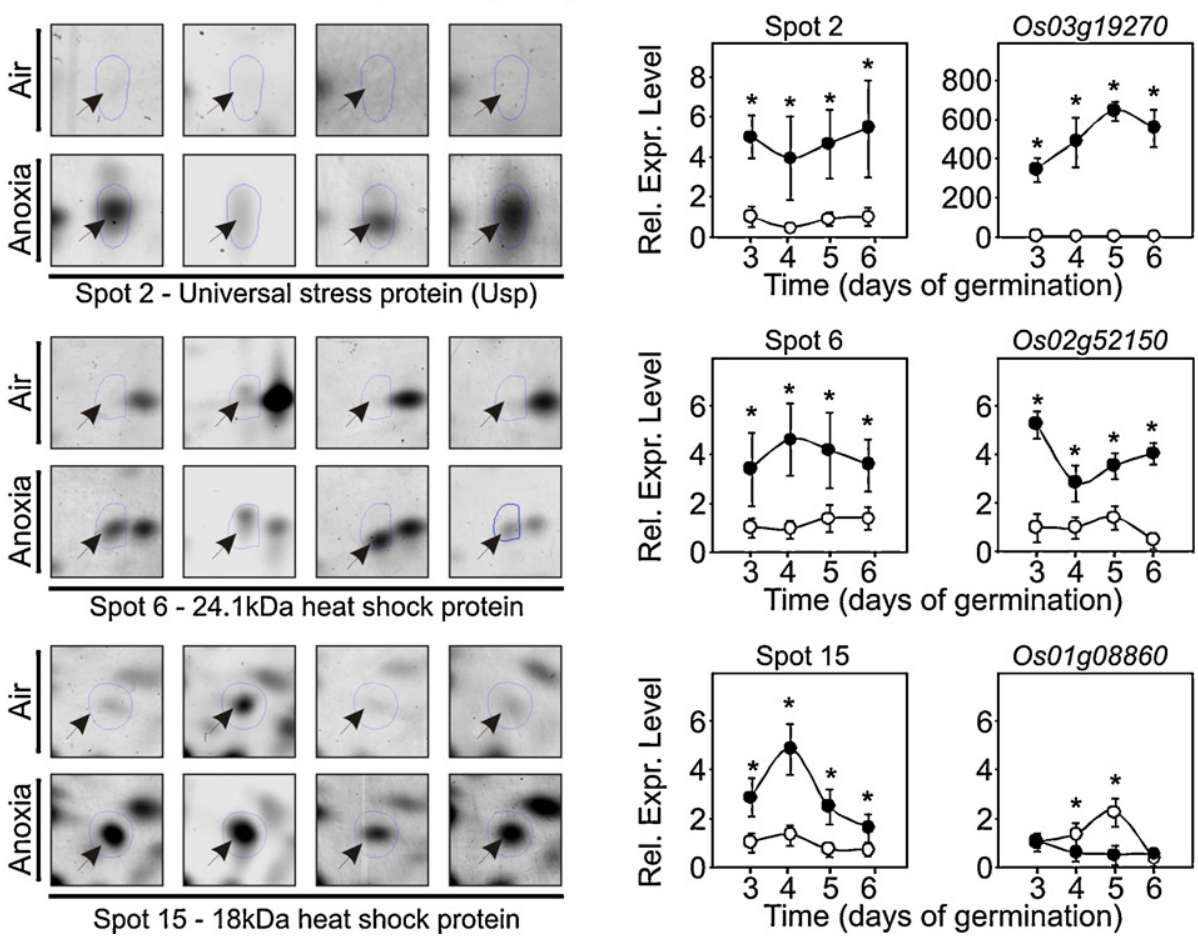

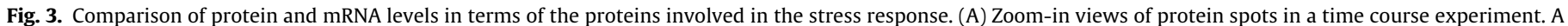

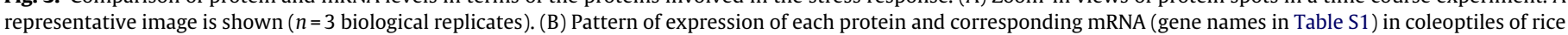

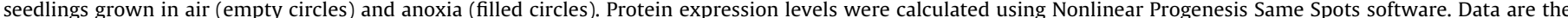

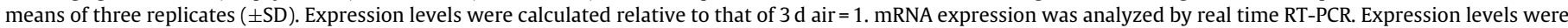

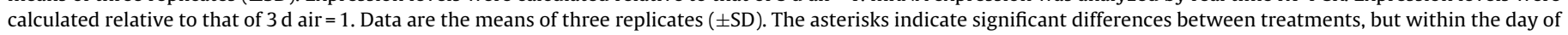
germination $(p<0.05)$. 

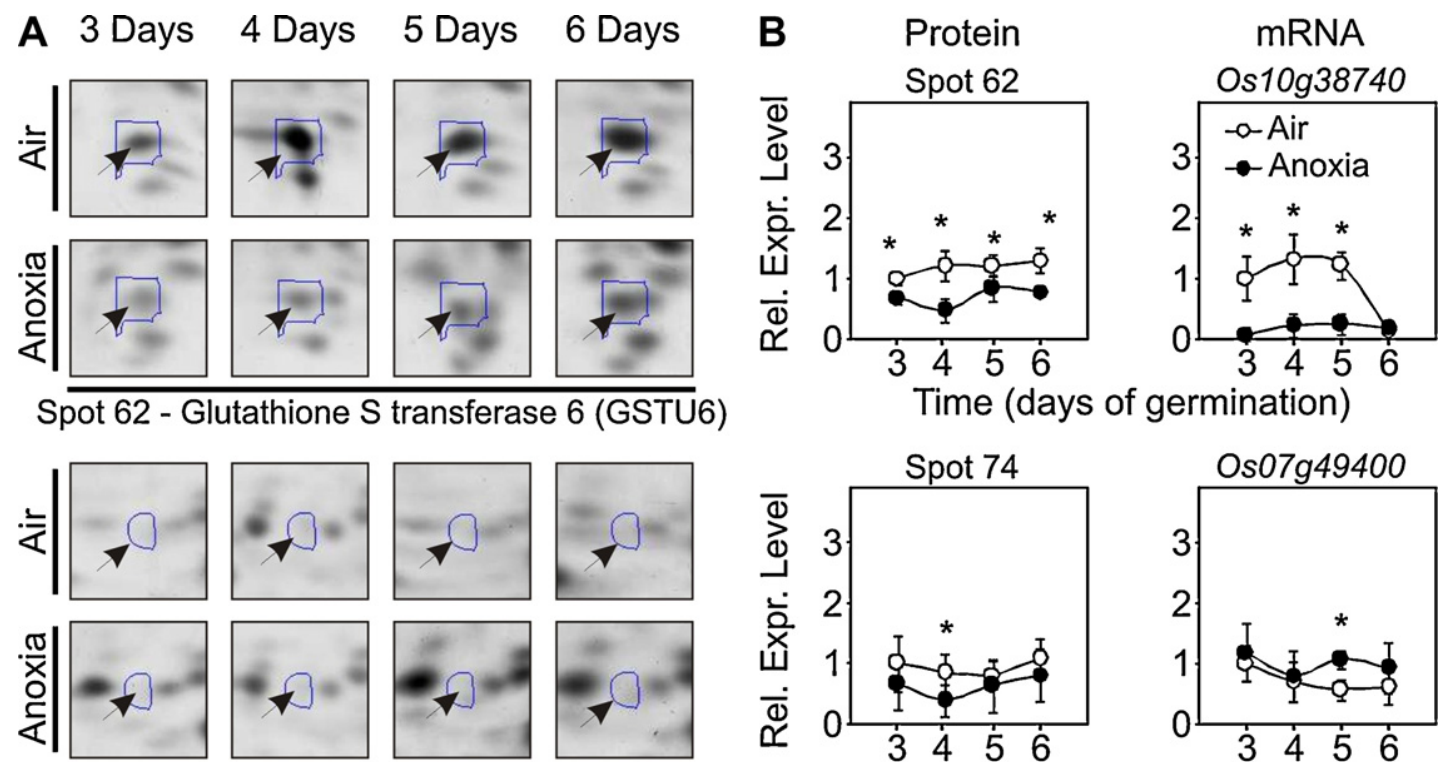

Spot 74 - L-ascorbate peroxidase 2 (cAPX2)
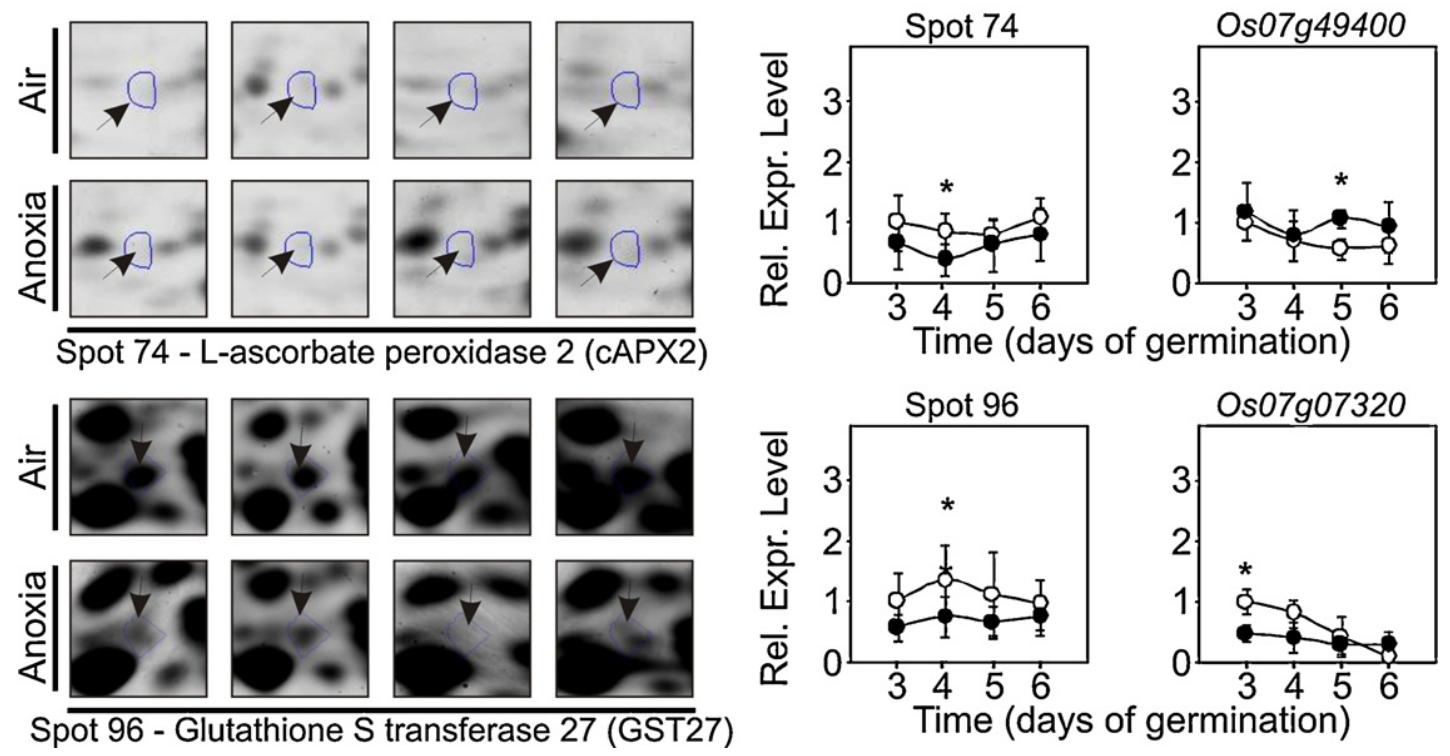

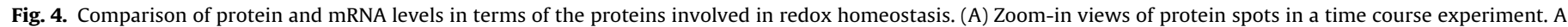

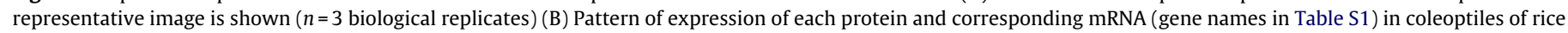

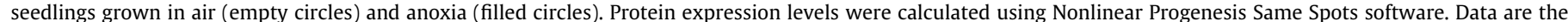

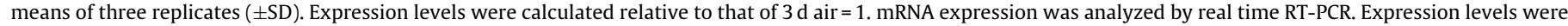

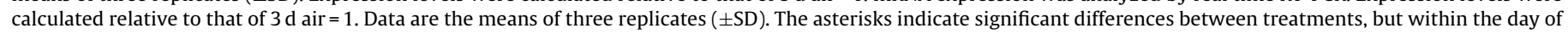
germination $(p<0.05)$.
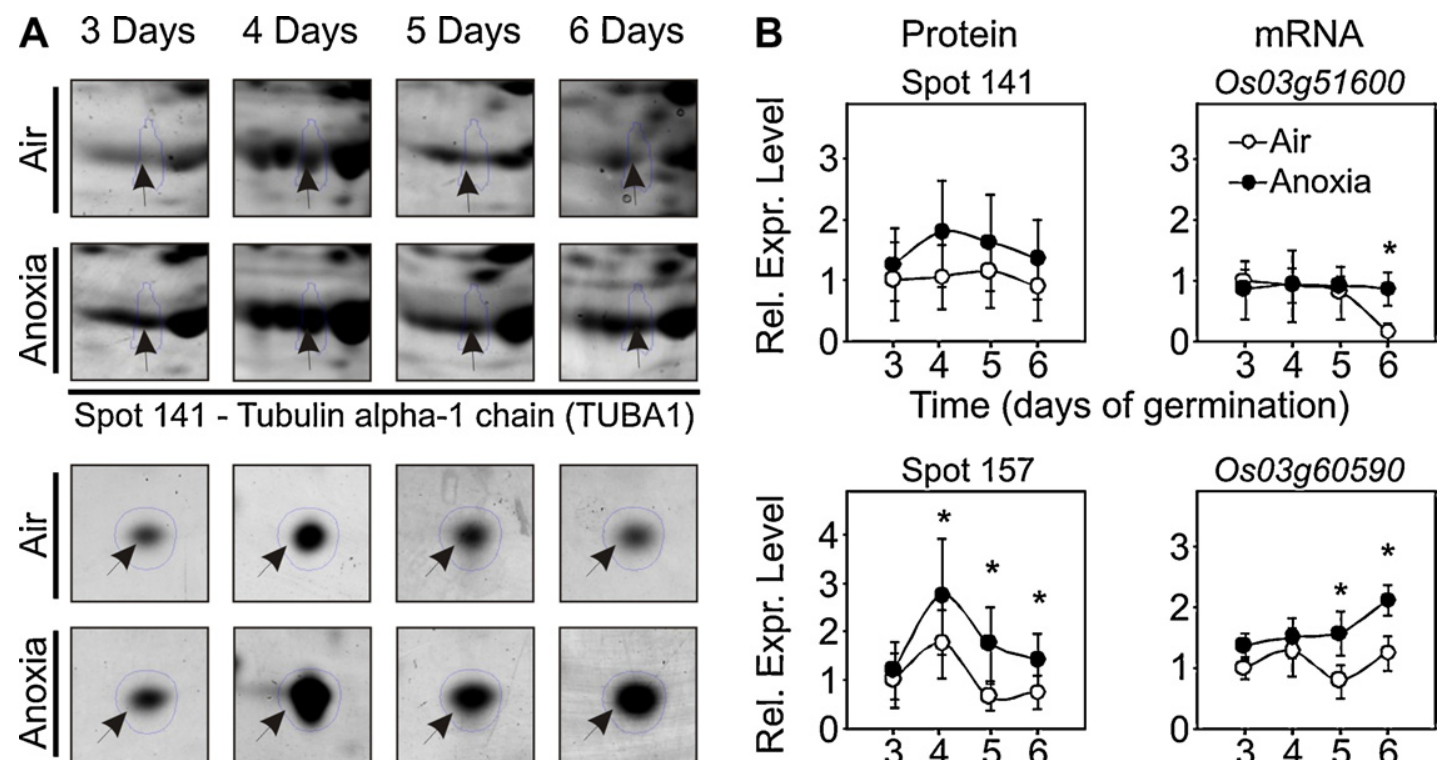

Spot 157 - Actin depolymerizing factor 4 (ADF4)

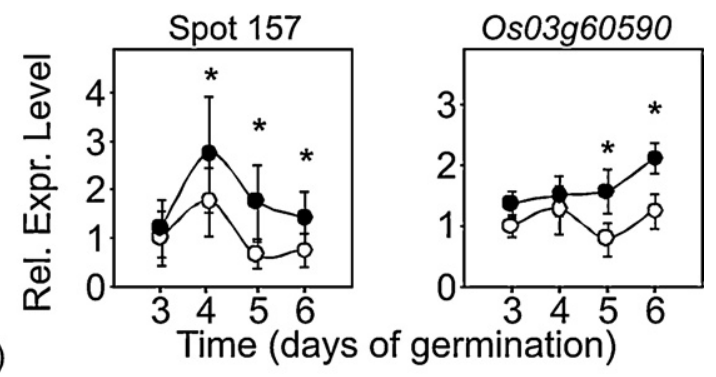

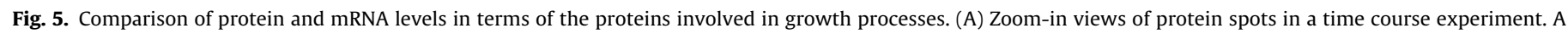

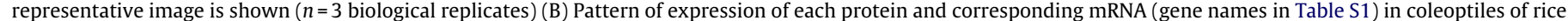

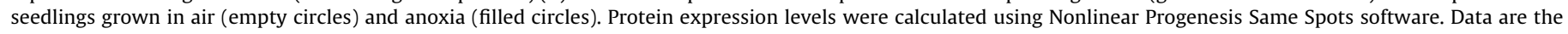

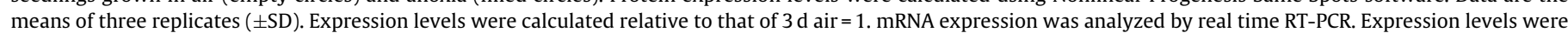

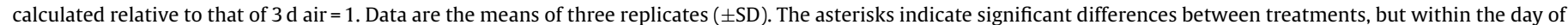
germination $(p<0.05)$. 
peroxidase (APX), superoxide dismutase, and catalase (Scandalios, 2002). Three spots (spots 62, 74, 96) were identified as ROS scavenger enzymes, namely glutathione S-transferase GSTU6 (spot 62), cytosolic L-ascorbate peroxidase cAPX2 (spot 74), and putative glutathione S-transferase GST27 (spot 96). All of these proteins were downregulated by anoxia (Fig. 4). Downregulation of cAPX2 under low oxygen is in accordance with the results obtained in soybean (Shi et al., 2008; Hashiguchi et al., 2009). In rice, the expression of APX is also downregulated after hydrogen peroxide treatment (Wan and Liu, 2008). A downregulation of APX and other ROS scavenger enzymes could increase $\mathrm{H}_{2} \mathrm{O}_{2}$ levels, which could act as signaling molecule under anoxia (Fukao and Bailey-Serres, 2004).

\section{Coleoptile growth}

Two anoxia-responsive proteins, namely a putative tubulin $\alpha-1$ chain (TUBA1, spot 141) and actin depolymerising factor 4 (ADF4; spot 157) are possibly involved in the fast growth of coleoptiles under anoxia.

Tubulin $\alpha-1$ chain (TUBA1; spot 141) was upregulated in response to anoxia, while its respective transcript remained unchanged except at day 6 (Fig. 5). There are two types of tubulins, $\alpha$-tubulins and $\beta$-tubulins, which collectively make microtubules. They play an important role in many plant cellular processes such as cell division, cell shape, and elongation (Mayer and Jürgens, 2002). Interestingly, rice roots, which are unable to grow under anoxia, show low levels of total $\beta$-tubulin mRNAs, whereas in coleoptiles, which are capable of anoxic elongation, high levels of total $\beta$ tubulin transcripts have been observed (Giani and Breviario, 1996). These results suggest that tubulins play a role in the anoxic growth of the rice coleoptile, although further experiments are required to establish the exact role of tubulins during the anoxic coleoptile growth.

Actin depolymerising factor 4 (ADF4; spot 157) was upregulated by anoxia both at protein and mRNA levels (Fig. 5). ADF4 is an actin-binding protein which is involved in the regulation of actin assembly (Augustine et al., 2008). Its expression has been induced in response to different stresses such as drought, salt and cold (Salekdeh et al., 2002; Yan et al., 2005; Ouellet et al., 2001). Huang et al. (2005) also observed the upregulation of an actin depolymerising factor, which is the only protein in common between our analysis and theirs. It is not surprising that most proteins do not match those identified by Huang et al. (2005), since our analysis used rice coleoptiles collected from seedlings grown in anoxia after seed imbibition. Since actin depolymerising factors promote the disaggregation of actin filaments, it is tempting to speculate that they may be involved in the fast anoxic growth of the rice coleoptile (Huang et al., 2005).

\section{Conclusions}

Rice is the only cereal crop that can germinate and grow in the complete absence of oxygen (Perata and Alpi, 1993; Magneschi and Perata, 2009). However, the growth is restricted to the coleoptile. Anoxia exerts a dramatic effect on the rice coleoptile transcriptome, with 1364 probe sets showing an increased expression and 1770 probe sets indicating a decreased expression, out of a total of about 22,500 probe sets on the Affymetrix ATH1 microarray (Lasanthi-Kudahettige et al., 2007). These numbers indicate that about $14 \%$ of transcripts change significantly under anoxia. Remarkably, although the amplitude of change was much lower, we found differential regulation in $14 \%$ of the protein spots. Although the microarray technology leads to a much wider exploration of gene expression at the level of transcripts than most proteomic approaches, our results suggest that, in rice coleoptiles, the mRNA changes observed are accompanied by similar changes in protein levels.

While some of the identified proteins confirmed the importance of known pathways for rice tolerance to anoxia (e.g. PDC, HSPs), other upregulated proteins provided new clues on the adaptation of rice coleoptiles to anoxia. The identification of CBL5 among the upregulated proteins means that CBL5 could be an excellent candidate for the CIPK15 partner, which has been shown to play an important role in rice tolerance to submergence, both at the germination and adult stages (Lee et al., 2009).

The identification of the Usp encoded by LOC_Os03g19270 suggested that this class of proteins may play an important role in plant adaptation to low oxygen. Although the function of Usps in plants is still obscure, it is interesting that they have been found to be upregulated by anoxia in rice (LOC_Os03g19270 in this study, and OsUsp1, Sauter et al., 2002), as well as in Arabidopsis (Mustroph et al., 2010). The mechanism(s) by which the anoxic coleoptile elongates longer than the aerobic coleoptile has remained elusive to date (Magneschi and Perata, 2009). In the reduced adh activity (rad) mutant in rice, elongation of the coleoptile is repressed under submergence suggesting that the fermentative metabolism is a prerequisite for coleoptile elongation (Matsumura et al., 1995). The increased expression of tubulin and of the ADF4 indicates that microtubules and actin filaments may play a role in the anoxic growth of the coleoptile. All of these hypotheses merit further experimental evaluation.

\section{Acknowledgment}

IS was supported by a PhD fellowship awarded by the Scuola Superiore Sant'Anna.

\section{Appendix A. Supplementary data}

Supplementary data associated with this article can be found, in the online version, at doi:10.1016/j.jplph.2011.07.009.

\section{References}

Ahsan N, Lee DG, Lee SH, Lee KW, Bahk JD, Lee BH. A proteomic screen and identification of waterlogging-regulated proteins in tomato roots. Plant Soil 2007;295:37-51.

Armstrong W. Aeration in higher plants. Adv Bot Res 1980;7:225-332.

Augustine RC, Vidali L, Kleinman KP, Bezanilla M. Actin depolymerizing factor is essential for viability in plants, and its phosphoregulation is important for tip growth. Plant J 2008;54:863-75.

Bailey-Serres J, Voesenek LA. Life in the balance: a signaling network controlling survival of flooding. Curr Opin Plant Biol 2010;13:489-94.

Banti V, Loreti E, Novi G, Santaniello A, Alpi A, Perata P. Heat acclimation and crosstolerance against anoxia in Arabidopsis. Plant Cell Environ 2008;31:1029-37.

Banti V, Mafessioni F, Loreti E, Alpi A, Perata P. The heat-inducible transcription factor HsfA2 enhances anoxia tolerance in arabidopsis. Plant Physiol 2010;152:1471-83.

Chang WWP, Huang L, Shen M, Webster C, Burlingame AL, Roberts JKM. Patterns of protein synthesis and tolerance of anoxia in root tips of maize seedlings acclimated to a low-oxygen environment, and identification of proteins by mass spectrometry. Plant Physiol 2000;122:295-317.

Fukao T, Bailey-Serres J. Plant responses to hypoxia - is survival a balancing act? Trends Plant Sci 2004;9:449-56.

Geigenberger P. Response of plant metabolism to too little oxygen. Curr Opin Plant Biol 2003;6:247-56.

Giani S, Breviario D. Rice $\beta$-tubulin mRNA levels are modulated during flower development and in response to external stimuli. Plant Sci 1996;116:147-57.

Glover T, Mitchell K. An introduction to biostatistics. McGraw Hill; 2002.

Guglielminetti L, Yamaguchi J, Perata P, Alpi A. Amylolytic activities in cereal seeds under aerobic and anaerobic conditions. Plant Physiol 1995;109:1069-76.

Hamilton EW, Heckathorn SA. Mitochondrial adaptations to $\mathrm{NaCl}$. Complex I is protected by anti-oxidants and small heat shock proteins, whereas complex II is protected by proline and betaine. Plant Physiol 2001;126:1266-74.

Hashiguchi A, Sakata K, Komatsu S. Proteome analysis of early-stage soybean seedlings under flooding stress. J Proteome Res 2009;8:2058-69.

Huang S, Greenway H, Colmer TD, Millar AH. Protein synthesis by rice coleoptiles during prolonged anoxia: implications for glycolysis, growth and energy utilization. Ann Bot 2005;96:703-15. 
Kolukisaoglu Ü, Weinl S, Blazevic D, Batistic O, Kudla J. Calcium sensors and their interacting protein kinases: genomics of the arabidopsis and rice CBL-CIPK signaling networks. Plant Physiol 2004;134:43-58.

Kong FJ, Oyanagi A, Komatsu S. Cell wall proteome of wheat roots under flooding stress using gel-based and LC MS/MS-based proteomics approaches. Biochim Biophys Acta 2010;1804:124-36.

Lasanthi-Kudahettige R, Magneschi L, Loreti E, Gonzali S, Licausi F, Novi G, et al. Transcript profiling of the anoxic rice coleoptile. Plant Physiol 2007;144:218-31.

Lee GJ, Roseman AM, Saibil HR, Vierling E. A small heat shock protein stably binds heat-denatured model substrates and can maintain a substrate in a foldingcompetent state. EMBO J 1997;16:659-71.

Lee BH, Won SH, Lee HS, Miyao M, Chung WI, Kim IJ, et al. Expression of the chloroplast-localized small heat shock protein by oxidative stress in rice. Gene 2000;245:283-90.

Lee KW, Chen PW, Lu CA, Chen S, Ho THD, Yu SM. Coordinated responses to oxygen and sugar deficiency allow rice seedlings to tolerate flooding. Sci Signal 2009;2:ra61.

Licausi F, Perata P. Low oxygen signaling and tolerance in plants. Adv Bot Res 2009;50:139-98.

Loreti E, Poggi A, Novi G, Alpi A, Perata P. A genome-wide analysis of the effects of sucrose on gene expression in arabidopsis seedlings under anoxia. Plant Physiol 2005; $137: 1130-8$

Magneschi L, Perata P. Rice germination and seedling growth in the absence of oxygen. Ann Bot 2009;103:181-96.

Mayer U, Jürgens G. Microtubule cytoskeleton: a track record. Curr Opin Plant Biol 2002;5:494-501.

Matsumura H, Takano T, Yoshida KT, Takeda G. A rice mutant lacking alcohol dehydrogenase. Breed Sci 1995;45:365-7.

Millar AH, Trend AE, Heazlewood JL. Changes in the mitochondrial proteome during the anoxia to air transition in rice focus around cytochrome-containing respiratory complexes. J Biol Chem 2004;279:39471-8.

Mortz E, Krogh TN, Vorum H, Görg A. Improved silver staining protocols for high sensitivity protein identification using matrix-assisted laser desorption/ionization-time of flight analysis. Proteomics 2001;1:1359-63.

Mujer CV, Rumpho ME, Lin JJ, Kennedy RA. Constitutive and inducible aerobic and anaerobic stress proteins in the Echinochloa complex and rice. Plant Physiol 1993;101:217-26.

Mustroph A, Lee SC, Oosumi T, Zanetti ME, Yang H, Ma K, et al. Cross-kingdom comparison of transcriptomic adjustments to low oxygen stress highlights conserved and plant-specific responses. Plant Physiol 2010;152:1484-500.

Nakazono M, Tsuji H, Li Y, Saisho D, Arimura S, Tsutsumi N, et al. Expression of a gene encoding mitochondrial aldehyde dehydrogenase in rice increases under submerged conditions. Plant Physiol 2000;124:587-98.

Oakley BR, Kirsch DR, Morris NR. A simplified ultrasensitive silver stain for detecting proteins in polyacrylamide gels. Anal Biochem 1980;105:361-3.

Ouellet F, Carpentier É, Cope MJ, Monroy AF, Sarhan F. Regulation of a wheat actindepolymerizing factor during cold acclimation. Plant Physiol 2001;125:360-8.

Perata P, Alpi A. Ethanol metabolism in suspension cultured carrot cells. Physiol Plantarum 1991;82:103-8.

Perata P, Alpi A. Plant responses to anaerobiosis. Plant Sci 1993;93:1-17.
Perata P, Matsukura C, Vernieri P, Yamaguchi J. Sugar repression of a gibberellins-dependent signalling pathway in barley embryos. Plant Cell 1997;9: 2197-208.

Perata P, Loreti E, Guglielminetti L, Alpi A. Carbohydrate metabolism and anoxia tolerance in cereal grains. Acta Bot Neerl 1998;47:269-83.

Perkins DN, Pappin DJC, Creasy DM, Cottrell JS. Probability-based protein identification by searching sequence databases using mass spectrometry data. Electrophoresis 1999;20:3551-67.

Salekdeh GH, Siopongco J, Wade LJ, Ghareyazie B, Bennett J. Proteomic analysis of rice leaves during drought stress and recovery. Proteomics 2002;2:1131-45.

Sarkar NK, Kim YK, Grover A. Rice sHsp genes: genomic organization and expression profiling under stress and development. BMC Genomics 2009;10:393.

Sauter M, Rzewuski G, Marwedel T, Lorbiecke R. The novel ethylene-regulated gene OsUsp1 from rice encodes a member of a plant protein family related to prokaryotic universal stress proteins. J Exp Bot 2002;53:2325-31.

Scandalios JG. The rise of ROS. Trends Biochem Sci 2002;27:483-6.

Shevchenko A, Wilm M, Vorm O, Mann M. Mass spectrometric sequencing of proteins from silver-stained polyacrylamide gels. Anal Chem 1996;68:850-8.

Shi F, Yamamoto R, Shimamura S, Hiraga S, Nakayama N, Nakamura T, et al. Cytosolic ascorbate peroxidase 2 (cAPX 2) is involved in the soybean response to flooding. Phytochemistry 2008;69:1295-303.

Subbaiah CC, Zhang J, Sachs MM. Involvement of intracellular calcium in anaerobic gene expression and survival of maize seedlings. Plant Physiol 1994;105:369-76.

Sun W, Bernard C, Cotte BVD, Montagu MV, Verbruggen N. At-HSP17.6A, encoding a small heat-shock protein in Arabidopsis, can enhance osmotolerance upon overexpression. Plant J 2001;27:407-15.

Sun W, Montagu MV, Verbruggen N. Small heat shock proteins and stress tolerance in plants. Biochim Biophys Acta 2002;1577:1-9.

Tadege M, Brändle R, Kuhlemeier C. Anoxia tolerance in tobacco roots: effect of overexpression of pyruvate decarboxylase. Plant J 1998;14:327-35.

Timperio AM, Egidi MG, Zolla L. Proteomics applied on plant abiotic stresses: role of heat shock proteins (HSP). J Proteomics 2008;71:391-411.

Tsuji H, Meguro N, Suzuki Y, Tsutsumi N, Hirai A, Nakazono M. Induction of mitochondrial aldehyde dehydrogenase by submergence facilitates oxidation of acetaldehyde during re-aeration in rice. FEBS Lett 2003;546:369-73.

Umeda M, Uchimiya H. Differential transcript levels of genes associated with glycolysis and alcohol fermentation in rice plants (Oryza sativa L.) under submergence stress. Plant Physiol 1994;106:1015-22.

Wan XY, Liu JY. Comparative proteomics analysis reveals an intimate protein network provoked by hydrogen peroxide stress in rice seedling leaves. Mol Cell Proteomics 2008;7:1469-88.

Yamauchi M, Aragones DV, Casayuran PR, Cruz PCS, Asis CA, Cruz RT. Seedling establishment and grain yield of tropical rice sown in puddled soil. Agron J 2000;92:275-82.

Yan S, Tang Z, Su W, Sun W. Proteomic analysis of salt stress-responsive proteins in rice root. Proteomics 2005;5:235-44.

Yang Q, Wang Y, Zhang J, Shi W, Qian C, Peng X. Identification of aluminumresponsive proteins in rice roots by a proteomic approach: cysteine synthase as a key player in $\mathrm{Al}$ response. Proteomics 2007;7:737-49. 